\title{
The Effect of Creatine Monohydrate Supplementation on Fatigue in Multiple Sclerosis
}

Maryam Abolhasani ${ }^{1,3}$, Soodeh Razeghi Jahromi ${ }^{2,3}$, Mansoureh Togha ${ }^{4}$, Mohammad Ali Sahraian ${ }^{3}$, Solmaz $^{*}$ Sadr-Jahani ${ }^{4}$

${ }^{1}$ Sports Medicine Research Center, Neuroscience Institute, Sina Hospital, Tehran University of Medical Sciences, Tehran, Iran. ${ }^{2}$ Department of Clinical Nutrition and Dietetics, Faculty of Nutrition and Food Technology, Shahid Beheshti University of Medical Sciences, Tehran, Iran.

${ }^{3}$ Multiple Sclerosis Research Center, Neuroscience Institute, Sina Hospital, Tehran University of Medical Sciences, Tehran, Iran.

${ }^{4}$ Iranian Center of Neurological Research, Neuroscience Institute, Tehran University of Medical Sciences, Tehran, Iran.

\section{A BSTRACT}

Introduction: Fatigue is considered to be one of the main causes of impaired quality of life among patients with multiple sclerosis (MS). Creatine enhances ATP synthesis and possesses antioxidant properties. There has been interest in the use of creatine supplement for fatigue and muscle strength in neurological disorders. It has previously been reported that, using 20 gram/day creatine supplement for 5-7 days followed by 5 gram/day for another 7 days did not ameliorate fatigue in patients with MS. In this study, we aimed to determine whether longer application of creatine supplement would be effective in controlling fatigue in these patients. Materials and Methods: In a double-blind controlled trial, 20 patients with MS with fatigue and mild to moderate disability were randomly assigned to creatine (10 gram, two times a day for 14 days) and placebo (10 patients in each group). Fatigue was assessed at baseline and after 14 days using fatigue severity scale (FSS). Results: No significant differences were observed between FSS score before and after the intervention. Furthermore, no significant difference was observed between the experimental groups in the mean difference of FSS. Conclusion: Creatine supplementation had no significant effect on fatigue score even after longer administration.

\section{Key words:}

1. Creatine

2. Fatigue

3. Multiple Sclerosis

4. Nervous System

Diseases

5. Dietary Supplements

* Corresponding Author: Soodeh Razeghi Jahromi

E-mail:Razeghi@sina.tums.ac.ir 


\title{
اثر مكمل كراتين مونوهيدرات بر خستگى در مالتييل اسكلروز
}

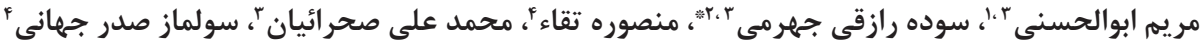

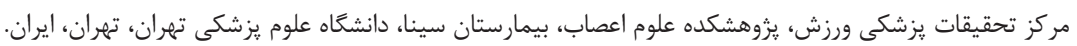

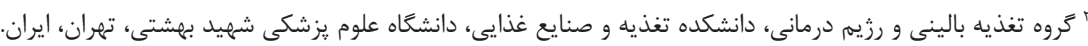

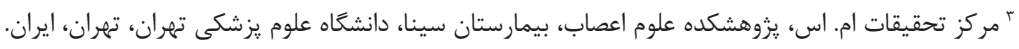

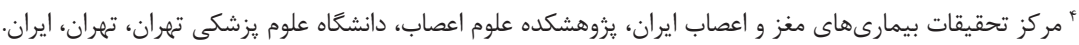

كليد وازهها:

ا

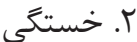

". مالتييل اسكلروز

أ. بيمارىهاى سيستم

عصبى

ه. مكمل هاى غذايى
مقدمه: خستخى به عنوان يكى از عوامل اصلى اختلال در كيفيت زندگى در بيماران مبتلا به مالتييل

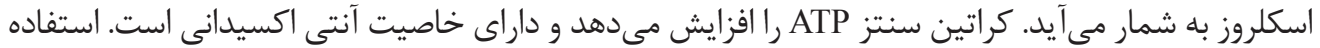

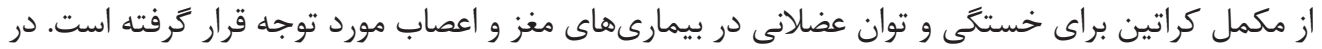

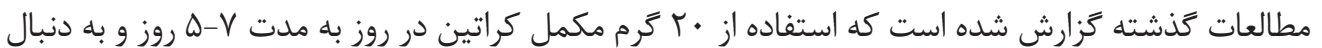

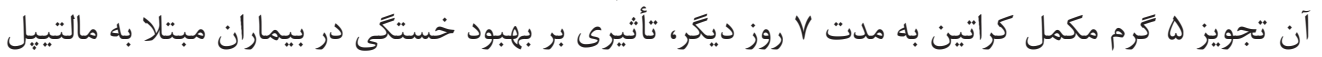

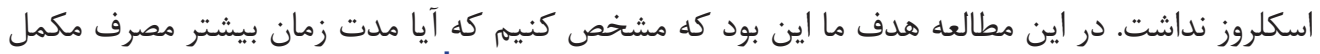

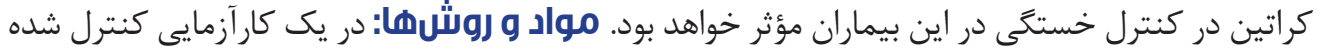

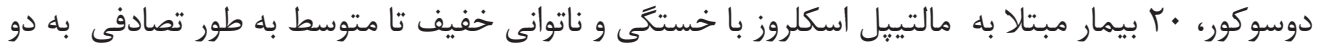

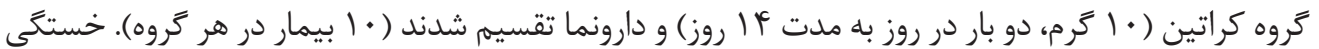

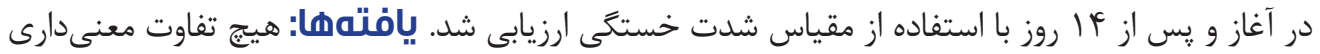

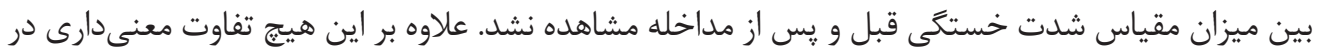

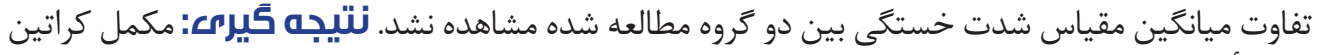

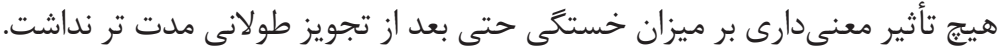

" نويسنده مسئول: سوده رازقى جهرمى آنى آدرس الكترونيكى: Razeghi@sina.tums.ac.ir 


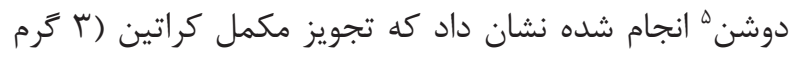

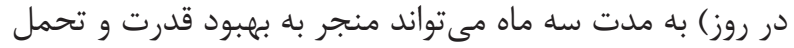

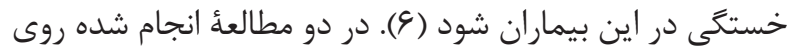

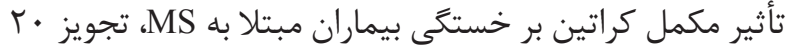

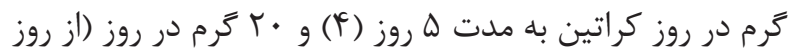

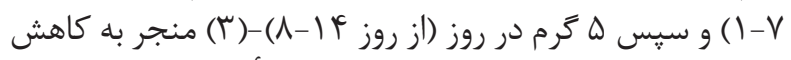

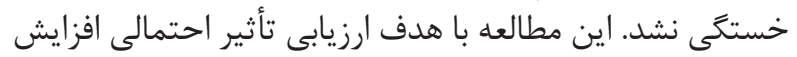

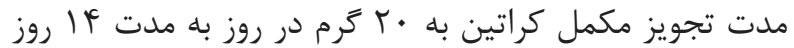

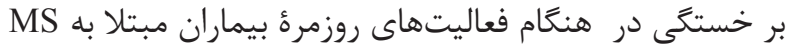

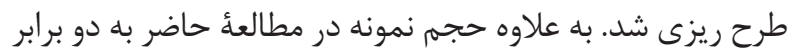
حجم نمونه در مطالعات بِيشين افزايش ياند دئ.

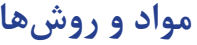

اين مطالعه به صورت كارآزمايى بالينى تصادفى دو سو كور

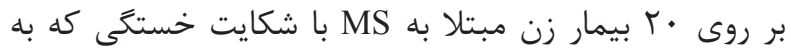

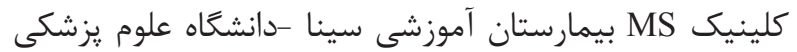

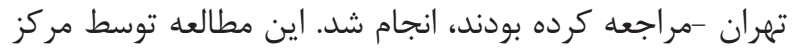

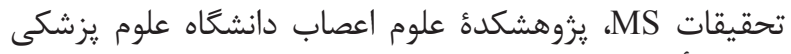

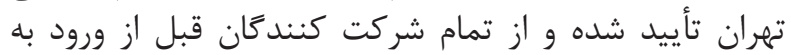
مطالعه رضايت نامهٔ كتبى اخذ كران تمرديد.

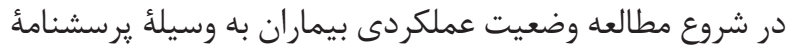
EDSS

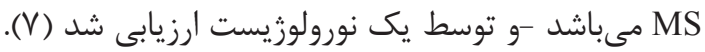

يرسشنامه EDSS يك مقياس بالينى و بر اساس يك معاينه

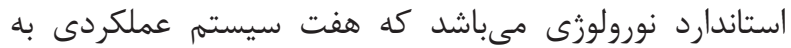

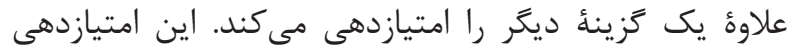

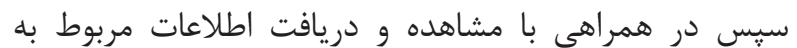

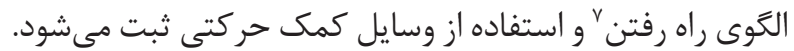

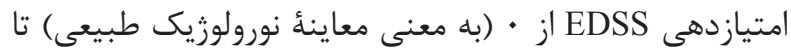

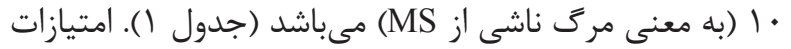

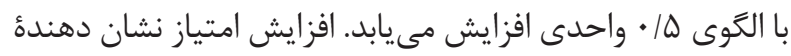
افزايش ميزان ناتوانى فرد است إنى

با توجه به اينكه امتياز ه/و يا كمتر براى افرادى در نظر كرفتئه

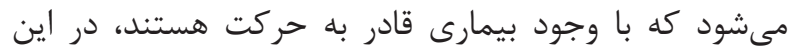

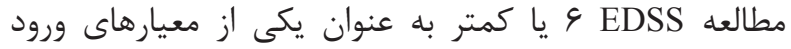

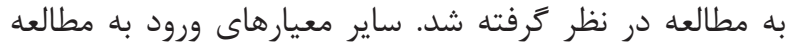

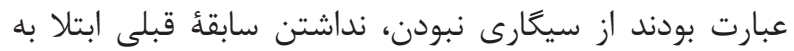

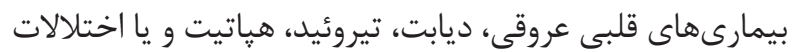

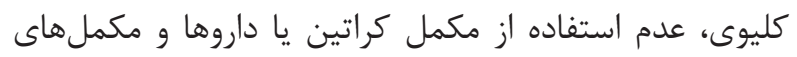

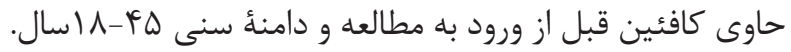

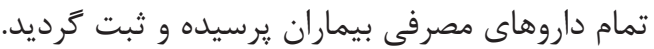
يرسشنامة FSS، مراى ارزيابى خستخى علامت دار استفاده

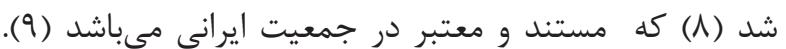

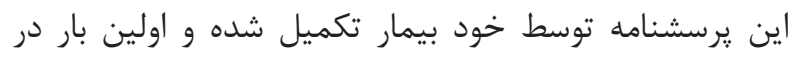

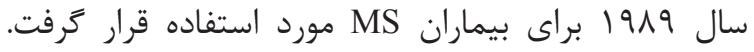

\footnotetext{
${ }^{1}$ Multiple sclerosis (MS)

${ }^{2}$ Phosphate creatine (PCr)

${ }^{3}$ Adenosine Triphosphate (ATP)

${ }^{4}$ Adenosine Diphosphate (ADP)
}

مالتيِّل اسكلروز (MS)' يك بيمارى نورولوزيك است كه ماده

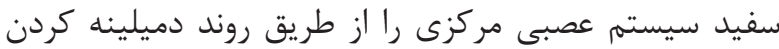
تحت تأثير قرار داده و به اين ترتيب عملكرد عضلات رات را مختل كرئل

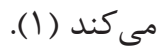

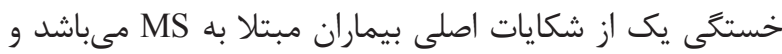

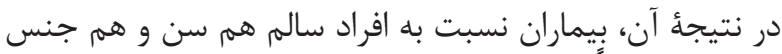

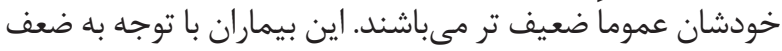
و خستگى تحمل كمترى نسبت به انجام فعاليتهاى بدن بدنى دارند

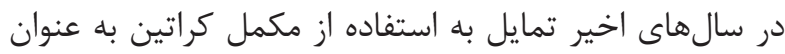

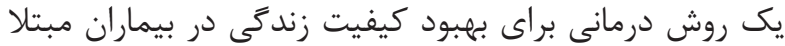

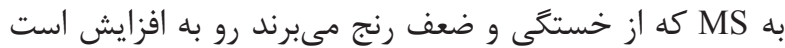

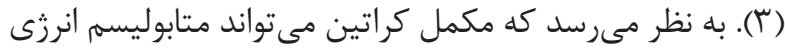

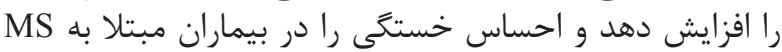

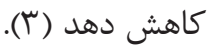

كراتين يك يروتئين غيرضرورى است و به طور ماتهو معمول در

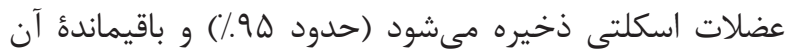

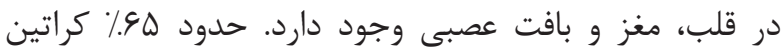

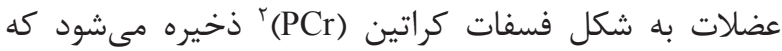

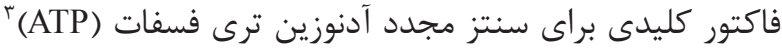

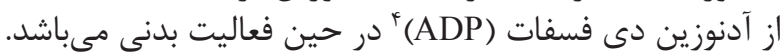

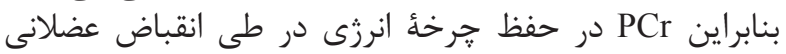

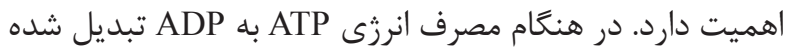

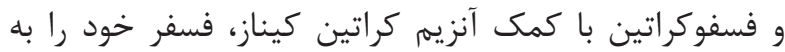

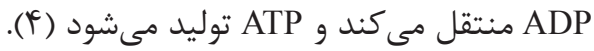

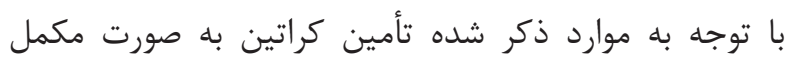

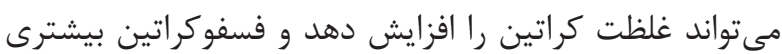

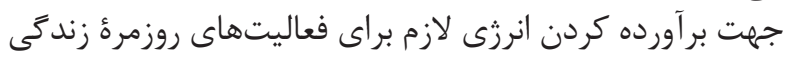

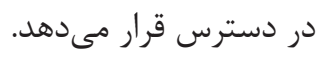

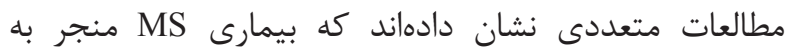

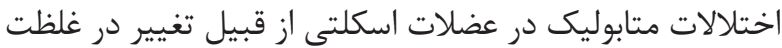
فسفات، PCr و آنزيمهاى اكسيداتيو دات در حين ورزش دات در مقايس

با افراد سالم مىشود (بات).

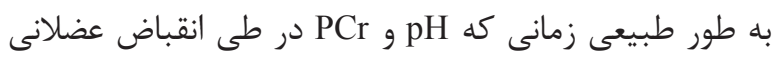

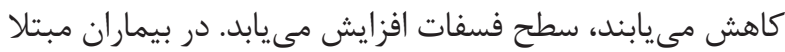

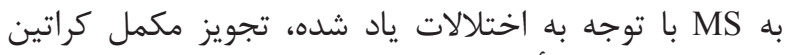

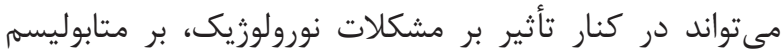

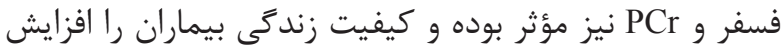

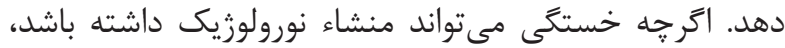

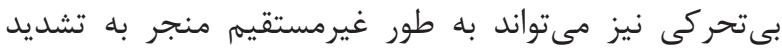

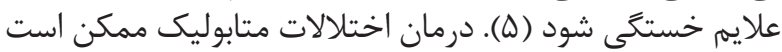

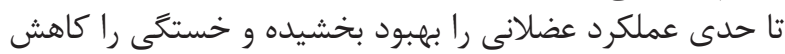

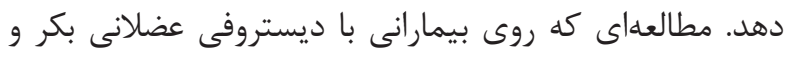

\footnotetext{
${ }^{5}$ Becker and Duchenne muscular dystrophy

${ }^{6}$ Krutzke's expand disability status score (EDSS)

${ }^{7}$ Gait

${ }^{8}$ Fatigue severity scale (FSS)
} 
جدول ا- يرسشنامئ EDSS (V).

\begin{tabular}{|c|c|}
\hline & $\begin{array}{l}\text { Pyramidal Functions } \\
0 \text { - Normal } \\
1 \text { - Abnormal signs without disability } \\
2 \text { - Minimal disability } \\
3 \text { - Mild to moderate paraparesis or hemiparesis (detectable weakness but most function sustained for short periods, } \\
\quad \text { fatigue a problem); severe monoparesis (almost no function) } \\
4 \text { - Marked paraparesis or hemiparesis (function is difficult), moderate quadriparesis (function is decreased but can } \\
\text { be sustained for short periods); or monoplegia } \\
5 \text { - Paraplegia, hemiplegia, or marked quadriparesis } \\
\text { - Quadriplegia } \\
9 \text { - (Unknown) }\end{array}$ \\
\hline व & $\begin{array}{l}\text { Cerebellar Functions } \\
0 \text { - Normal } \\
1 \text { - Abnormal signs without disability } \\
2 \text { - Mild ataxia (tremor or clumsy movements easily seen, minor interference with function) } \\
3 \text { - Moderate truncal or limb ataxia (tremor or clumsy movements interfere with function in all spheres) } \\
4 \text { - Severe ataxia in all limbs (most function is very difficult) } \\
5 \text {-Unable to perform coordinated movements due to ataxia } \\
9 \text { - (Unknown) } \\
\quad \text { Record \#1 in small box when weakness (grade } 3 \text { or worse on pyramidal) interferes with testing. }\end{array}$ \\
\hline & $\begin{array}{l}\text { Brain Stem Functions } \\
0 \text { - Normal } \\
1 \text { - Signs only } \\
2 \text { - Moderate nystagmus or other mild disability } \\
3 \text { - Severe nystagmus, marked extraocular weakness, or moderate disability of other cranial nerves } \\
4 \text { - Marked dysarthria or other marked disability } \\
5 \text { - Inability to swallow or speak } \\
9 \text { - (Unknown) }\end{array}$ \\
\hline & $\begin{array}{l}\text { Sensory Functions } \\
0 \text { - Normal } \\
1 \text { - Vibration or figure-writing decrease only in one or two limbs } \\
2 \text { - Mild decrease in touch or pain or position sense, and/or moderate decrease in vibration in one or two limbs; or vibratory (c/s } \\
\text { figure writing) decrease alone in three or four limbs } \\
3 \text { - Moderate decrease in touch or pain or position sense, and/or essentially lost vibration in one or two limbs; or mild decrease in } \\
\text { touch or pain and/or moderate decrease in all proprioceptive tests in three or four limbs } \\
\text { - Marked decrease in touch or pain or loss of proprioception, alone or combined, in one or two limbs; or moderate decrease in } \\
\text { touch or pain and/or severe proprioceptive decrease in more than two limbs } \\
5 \text { - Loss (essentially) of sensation in one or two limbs; or moderate decrease in touch or pain and/or loss of proprioception for } \\
\text { most of the body below the head } \\
6 \text { - Sensation essentially lost below the head } \\
9 \text { - (Unknown) }\end{array}$ \\
\hline$\square$ & $\begin{array}{l}\text { Bowel and Bladder Functions } \\
\text { (Rate on the basis of the worse function, either bowel or bladder) } \\
0 \text { - Normal } \\
1 \text { - Mild urinary hesitance, urgency, or retention } \\
2 \text { - Moderate hesitance, urgency, retention of bowel or bladder, or rare urinary incontinence (intermittent self- } \\
\text { catheterization, manual compression to evacuate bladder, or finger evacuation of stool) } \\
3 \text { - Frequent urinary incontinence } \\
4 \text { - In need of almost constant catheterization (and constant use of measures to evacuate stool) } \\
5 \text { - Loss of bladder function } \\
6 \text { - Loss of bowel and bladder function } \\
9 \text { - (Unknown) }\end{array}$ \\
\hline प & $\begin{array}{l}\text { Visual Functions } \\
0 \text { - Normal } \\
1 \text { - Scotoma with visual acuity (corrected) better than } 20 / 30 \\
2 \text { - Worse eye with scotoma with maximal visual acuity (corrected) of } 20 / 30-20 / 59 \\
3 \text { - Worse eye with large scotoma, or moderate decrease in fields, but with maximal visual acuity (corrected) of 20/60-20/99 } \\
4 \text { - Worse eye with marked decrease of fields and maximal visual acuity (corrected) of } 20 / 100-20 / 200 \text {; grade } 3 \text { plus maximal } \\
\quad \text { acuity of better eye of } 20 / 60 \text { or less } \\
5 \text { - Worse eye with maximal visual acuity (corrected) less than } 20 / 200 \text {; grade } 4 \text { plus maximal acuity of better eye of } 20 / 60 \text { or less } \\
6 \text { - Grade } 5 \text { plus maximal visual acuity of better eye of } 20 / 60 \text { or less } \\
9 \text { - (Unknown) } \\
\quad \text { Record \#1 in small box for presence of temporal pallor }\end{array}$ \\
\hline & $\begin{array}{l}\text { Cerebral (or Mental) Functions } \\
0 \text { - Normal } \\
1 \text { - Mood alteration only (does not affect EDSS score) } \\
2 \text { - Mild decrease in mentation } \\
3 \text { - Moderate decrease in mentation } \\
4 \text { - Marked decrease in mentation (chronic brain syndrome - moderate) } \\
5 \text { - Dementia or chronic brain syndrome - severe or incompetent } \\
9 \text { - (Unknown) }\end{array}$ \\
\hline
\end{tabular}




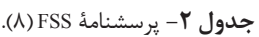

\begin{tabular}{|c|c|}
\hline سؤال & \\
\hline وقتى خستـه مىشوم، انكَيزهُ من كمتر است. & 1 \\
\hline ورزش موجب خستخى من مىشود. & $r$ \\
\hline من به راحتى خسته مىشوم. & r \\
\hline خستكى در فعاليتهاى بدنى من اختلال ايجاد مى كند. & r \\
\hline خستگى مشكلات متعددى براى من ايجاد مى كند. & $\Delta$ \\
\hline خستكى مانع از فعاليت مداوم بدنى من مىشود. & \& \\
\hline خستكَى در انجام وظايف و مسئوليتهاى اصلى من اختلال ايجاد مى كند. & V \\
\hline خستگى يكىى از سه نشانئ بسيار ناتوان كنندهُ من مىباشد. & $\Lambda$ \\
\hline خستكى در كار، زندگى خانوادگى و اجتماعى من اختلال ايجاد مى كند. & 9 \\
\hline
\end{tabular}

مطالعه تكميل نمودند. آناليز آمارى

دادهها با استفاده از نرم افزار آمارى 17 SPSS Version مورد

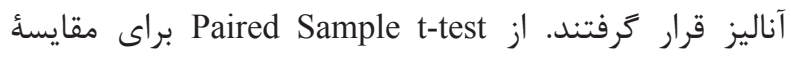

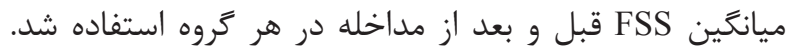

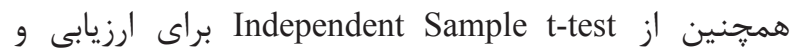

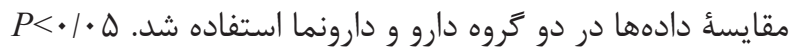

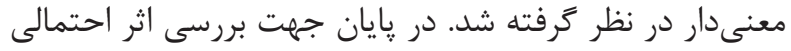

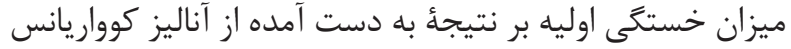

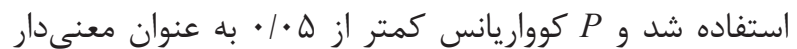

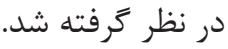

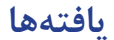

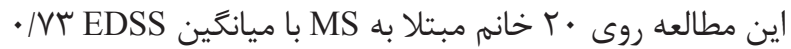

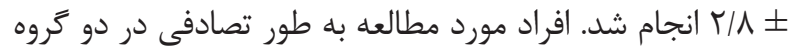

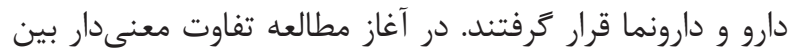

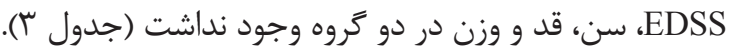
از يرسشنامة FSS براى ارزيابى خستخى استفاده شد. يرسشنامه

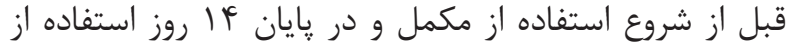

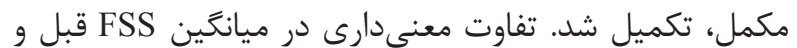

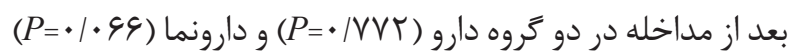

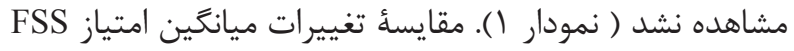

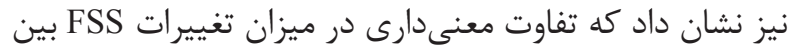

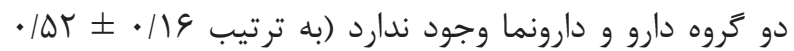

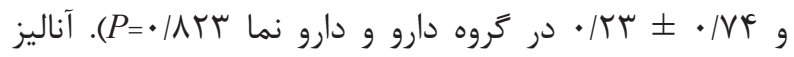

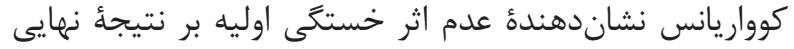

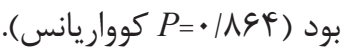

اين يرسشنامه به طور اختصاصى خستكى را اندازه مى كيرد و

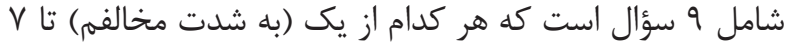

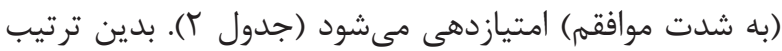

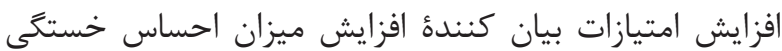

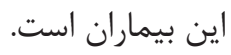

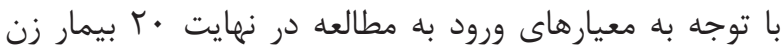

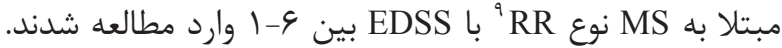

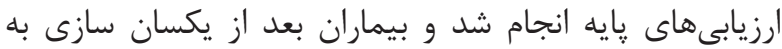
صورت تصادفى در دو كروه دارو و دارونما قرار كرفتنند.

براى گروه دارو شامل · ل بيمار، مكمل يودر كراتين مونوهيدرات

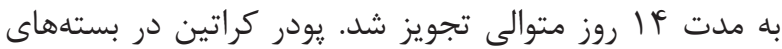

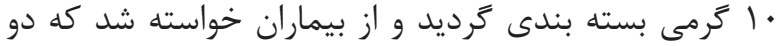

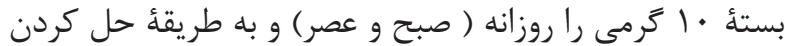

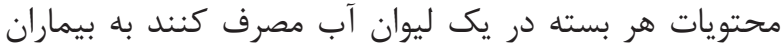

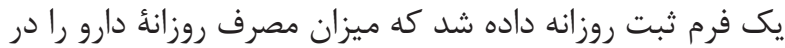

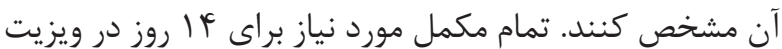

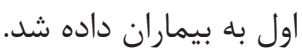

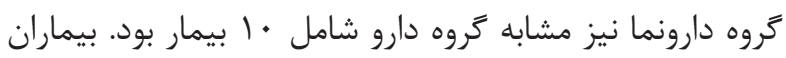

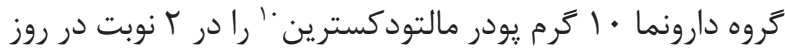
و به مدت أ أ روز متوالى استفاده كردند. شركت كنندكان موافقت كردند كه از يك هفته قبل از مطالعه

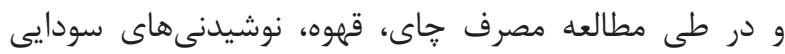

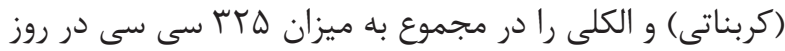

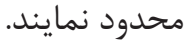

شركت كنندًان يكبار قبل از مطالعه به جهت به به دست آوردن

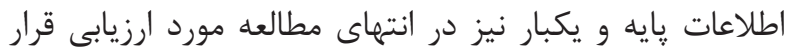

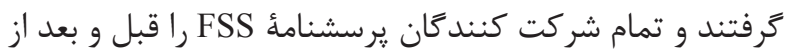




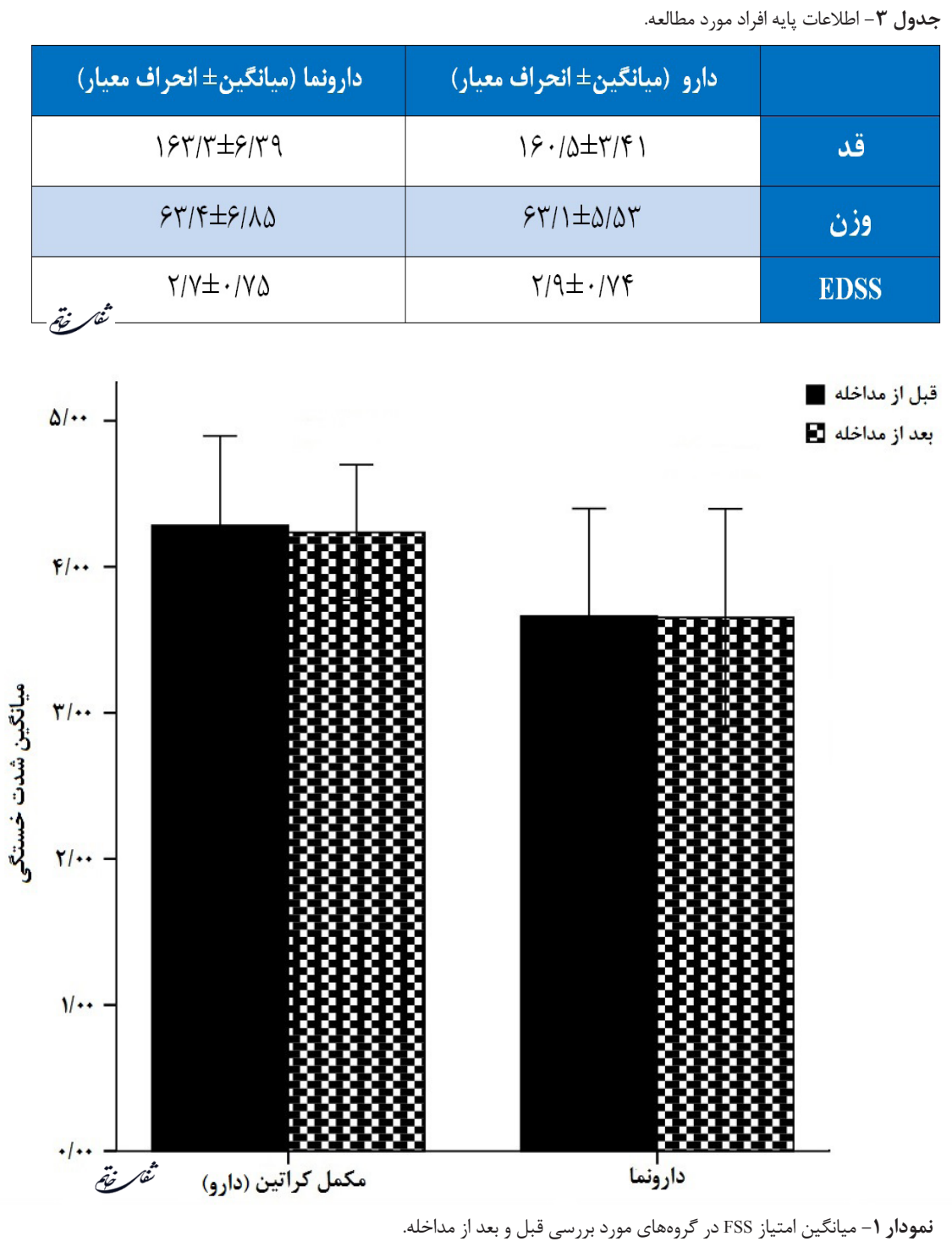

Martin و Tarnopolsky به مدت بيش از • ا روز در افراد مبتلا به ديستروفى منجر بن داديه

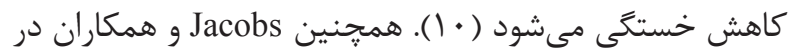

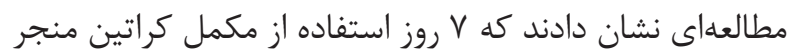

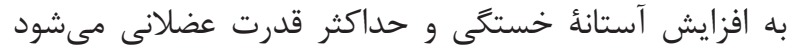

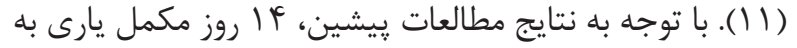

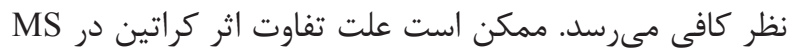

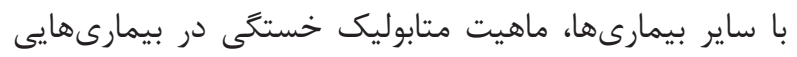

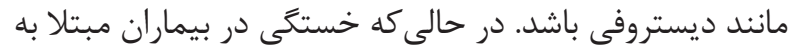
ماهيت نورولوزيك نيز دارد.

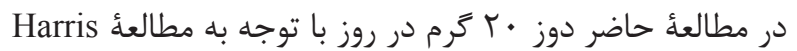

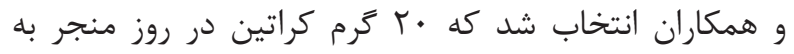

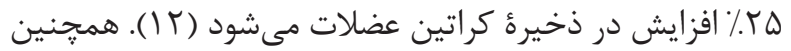
Mazzini

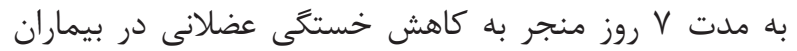

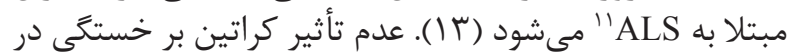

بحث و نتيجه گَيرى

مطالعهُ فوق بر اساس اين فرضيه انجام شد كه مكمل كراتين

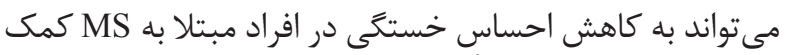

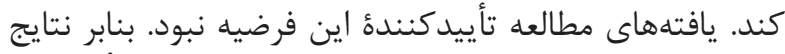

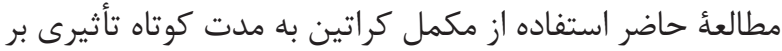

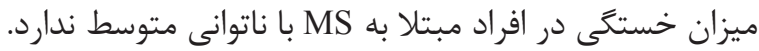
يافته هاى مطالعهُ حاضر همسو با نتايج مطالعه

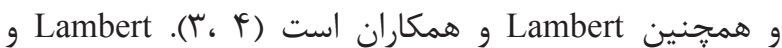

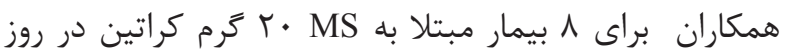

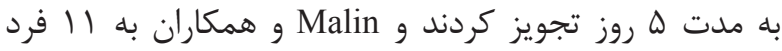

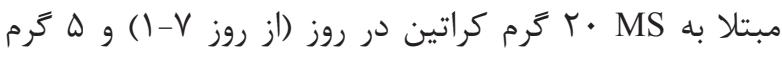

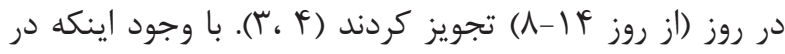

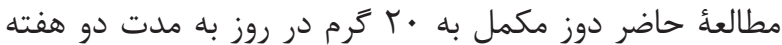

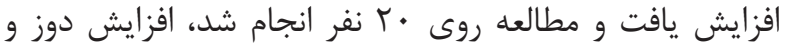

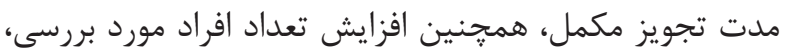

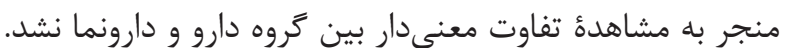

${ }^{11}$ Amyotrophic lateral sclerosis (ALS) 
نشد. مطالعات بيشين نشان دادهاند كه در افرادى كه بيشتر از كاز

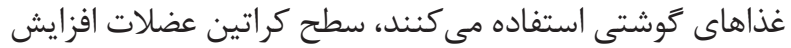

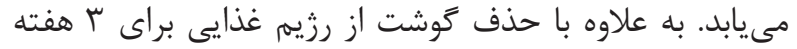

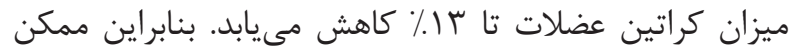

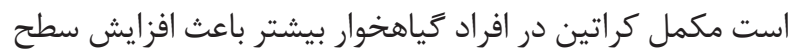

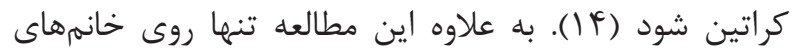

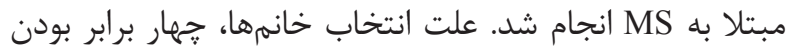
شيوع MS در خانمها نسبت به آقايان بود. افزايش مدت مصرف مكمل كراتين منجر به كاهش احساس

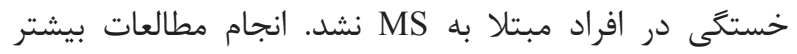

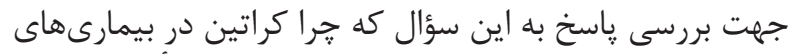

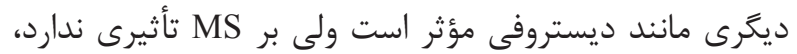

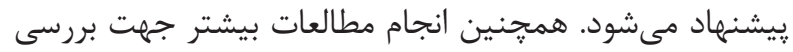

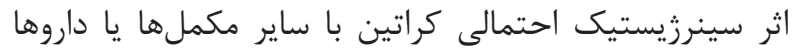

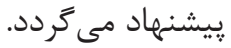

1. Razeghi Jahromi S, Toghae M, Razeghi Jahromi MJ, Aloosh M. Dietary pattern and risk of multiple sclerosis. Iran J Neurol. 2012; 11 (2): 47-53.

2. Forsberg A, Nilsagård Y, Boström K. Perceptions of using videogames in rehabilitation: a dual perspective of people with multiple sclerosis and physiotherapists. Disabil Rehabil. 2014; 1(10): 1-7.

3. Malin SK, Cotugna N, Fang CS. Effect of creatine supplementation on muscle capacity in individuals with multiple sclerosis. J Diet Suppl. 2008; 5(1): 20-32.

4. Lambert CP, Archer RL, Carrithers JA, Fink WJ, Evans WJ, Trappe TA. Influence of creatine monohydrate ingestion on muscle metabolites and intense exercise capacity in individuals with multiple sclerosis. Arch Phys Med Rehab. 2003; 84(8): 1206-10.

5. Motl RW, McAuley E, Snook EM, Gliottoni RC. Physical activity and quality of life in multiple sclerosis: intermediary roles of disability, fatigue, mood, pain, self-efficacy and social support. Psychol Health Med. 2009; 14(1): 111-24.

6. Louis M, Lebacq J, Poortmans JR, Belpaire-Dethiou MC, Devogelaer JP, Van Hecke P, et al. Beneficial effects of creatine supplementation in dystrophic patients. Muscle Nerve. 2003; 27(5): 604-10.

7. Kurtzke JF. Rating neurologic impairment in multiple sclerosis an expanded disability status scale (EDSS). Neurology. 1983; 33(11): 1444-52.

8. Krupp LB, LaRocca NG, Muir-Nash J, Steinberg AD.
بيماران مبتلا به MS مى بواند به علت نقص در عملكرد ناقلين

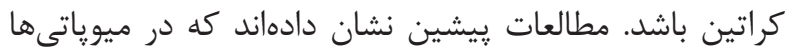

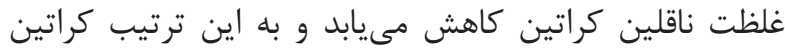

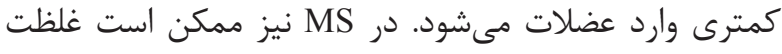

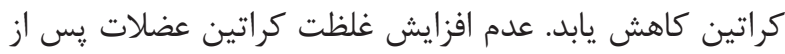

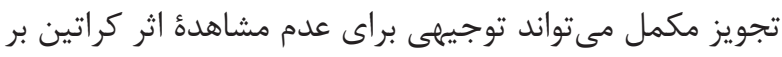

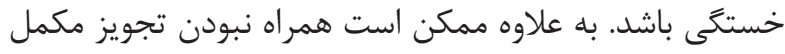

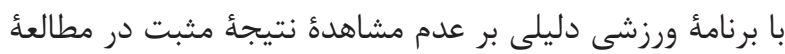

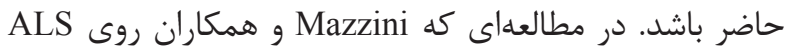

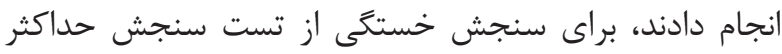

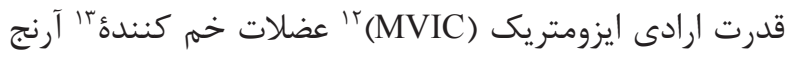

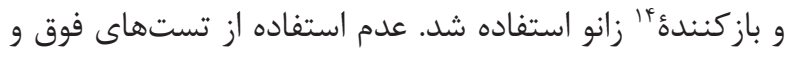

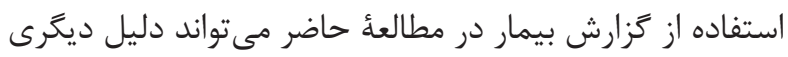

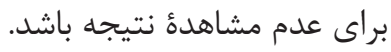

در مطالعه حاضر ميزان دريافت كراتين از طريق غذا محاسبه

The fatigue severity scale: application to patients with multiple sclerosis and systemic lupus erythematosus. Arch Neurol. 1989; 46(10): 1121-3.

9. Ghotbi N, Nakhostin N, Ansari SF, Aryan Shamili H, Choobsaz HM. Fatigue in Iranian patients with neurological conditions: an assessment with Persian fatigue severity scale. HSJ. 2013; 7 (4): 395-402.

10. Tarnopolsky M, Martin J. Creatine monohydrate increases strength in patients with neuromuscular disease. Neurology. 1999; 52(4): 854-7.

11. Jacobs PL, Mahoney ET, Cohn KA, Sheradsky LF, Green BA. Oral creatine supplementation enhances upper extremity work capacity in persons with cervicallevel spinal cord injury. Arch Phys Med Rehabil. 2002; 83(1): 19-23.

12. Harris RC, Soderlund K, Hultman E. Elevation of creatine in resting and exercised muscle of normal subjects by creatine supplementation. Clin Sci. 1992; 83(3): 367-74.

13. Mazzini L, Balzarini C, Colombo R, Mora G, Pastore I, De Ambrogio R, et al. Effects of creatine supplementation on exercise performance and muscular strength in amyotrophic lateral sclerosis: preliminary results. J Neurol Sci. 2001; 191(1): 139-44.

14. Lukaszuk JM, Robertson RJ, Arch JE, Moyna NM. Effect of a defined lacto-ovo-vegetarian diet and oral creatine monohydrate supplementation on plasma creatine concentration. J Strength Cond Res. 2005; 19(4): 735-40.

\footnotetext{
${ }^{12}$ Maximum voluntary isometric contraction (MVIC)

${ }^{13}$ Flexor

${ }^{14}$ Extensor
} 